\title{
Equations of Motion (EOM) Methods for Computing Electron Affinities
}

\author{
Jack Simons \\ Chemistry Department and Henry Eyring \\ Center for Theoretical Chemistry \\ University of Utah \\ Salt Lake City, Utah 84112
}

The ab initio calculation of molecular electron affinities (EA) is a difficult task because the energy of interest is a very small fraction of the total electronic energy of the parent neutral. That is, EAs typically lie in the 0.01-10 eV range, but the total electronic energy of even a small molecule is usually several orders of magnitude larger. Moreover, because the EA is an intensive quantity but the total energy is an extensive quantity, the difficulty in evaluating EAs to within a fixed specified (e.g., $\pm 0.1 \mathrm{eV}$ ) accuracy becomes more and more difficult as the size and number of electrons in the molecule grows. The situation becomes especially problematic when studying extended systems such as solids, polymers, or surfaces for which the EA is an infinitesimal fraction of the total energy. The equations of motion (EOM) methods offer a route to calculating the intensive EAs directly as eigenvalues of a set of working equations. A history of the development of equations of motion theories as applied to EAs, their numerous practical implementations, and their relations to Greens function or propagator theories are covered in this contribution. EOM methods based upon Møller-Plesset, Multiconfiguration self-consistent field, and coupled-cluster reference wave functions are included in the discussion as is the application of EOM methods to metastable resonance states of anions. 


\section{Background}

The vertical (i.e., at any given fixed molecular geometry) electron affinity (EA) of a molecule can be computed by (approximately) solving the Schrödinger equation for the energy $\mathrm{E}(0, \mathrm{~N})$ of the $\mathrm{N}$-electron neutral molecule and the Schrödinger equation for the energy $E(K, N+1)$ of the $K^{\text {th }}$ state of the $\mathrm{N}+1$-electron anion and subtracting the two energies:

$\mathrm{EA}=\mathrm{E}(0, \mathrm{~N})-\mathrm{E}(\mathrm{K}, \mathrm{N}+1)$

Here, we use $\mathrm{K}$ to label the electronic state of the anion that one wishes to study, and 0 to label the state of the neutral (usually but not necessarily the ground state) to which the electron is being attached.

In using such an approach to obtaining the EA, one is faced with a very difficult numerical challenge because both $\mathrm{E}(0, \mathrm{~N})$ and $\mathrm{E}(\mathrm{K}, \mathrm{N}+1)$ tend to be extremely large (negative) numbers, whereas EA nearly always lies in the range 0-10 eV. For example, the EA of the ${ }^{4} \mathrm{~S}_{3 / 2}$ state of the carbon atom is ${ }^{1} 1.262119 \pm 0.000020 \mathrm{eV}$, whereas the total electronic energy of this state of $\mathrm{C}$ is $-1030.080 \mathrm{eV}$ (relative to a $\mathrm{C}^{6+}$ nucleus and six electrons infinitely distant and not moving that defines the zero of energy). Since the EA is ca. $0.1 \%$ of the total energy of $\mathrm{C}$, one needs to compute the $\mathrm{C}$ and $\mathrm{C}^{-}$electronic energies to accuracies of $0.01 \%$ or better to calculate the EA to within $10 \%$.

This observation shows only the "tip of the iceberg", however as the major problem relates to the fact that $\mathrm{E}(0, \mathrm{~N})$ and $\mathrm{E}(\mathrm{K}, \mathrm{N}+1)$ are extensive properties whereas EA is an intensive quantity. For example, the EA of $\mathrm{C}_{2}$ in its $\mathrm{X}^{2} \Sigma_{\mathrm{g}}^{+}$ground electronic state is ${ }^{1} 3.269 \pm 0.006 \mathrm{eV}$ near the equilibrium bond length $\mathrm{R}_{\mathrm{e}}$ but only $1.2621 \mathrm{eV}$ at $\mathrm{R}$ $\rightarrow \infty$ (i.e., the same as the EA of a carbon atom). However, the total electronic energy of $\mathrm{C}_{2}$ is $-2060.160 \mathrm{eV}$ at $\mathrm{R} \rightarrow \infty$ and lower by ca. $3.6 \mathrm{eV}$ (the dissociation energy ${ }^{2}$ of $\mathrm{C}_{2}$ ) at $\mathrm{R}_{\mathrm{e}}$, so again EA is a very small fraction of the total energies. For buckyball $\mathrm{C}_{60}$, the EA is ${ }^{1}$ $2.666 \pm 0.001 \mathrm{eV}$, but the total electronic energy is sixty times $-1030.080 \mathrm{eV}$ minus the atomization energy (i.e., the energy change for $\mathrm{C}_{60} \rightarrow 60 \mathrm{C}$ ) of this compound. Clearly, the challenge of evaluating EA to within even $50 \%$ becomes more and more difficult as 
the size (i.e., number of electrons) in the molecule grows, and it becomes impossible when the system of interest is an infinite solid, surface, or polymer. This same kind of difficulty (i.e., calculating an intensive quantity as the difference between to extensive energies) plagues the computation of EAs and of ionization potentials (IPs), bond energies, and electronic excitation energies.

Of course, much progress can be made in computing EAs as differences between anion and neutral total energies ${ }^{3}$ because of large systematic cancellation in energy errors. For example, the pair correlation energies of the two 1s electron pairs in $\mathrm{C}_{2}$ is quite large, but is very nearly the same as in $\mathrm{C}_{2}^{-}$, so even a large percent error made in computing these contributions to the total energy may not greatly affect the EA computed by subtracting $E(K, N+1)$ from $E(0, N)$. Nevertheless, in the late 1960 s and early 1970 s, workers were motivated to develop methods that would allow intensive energy differences such as EAs, ionization potentials (IPs) and excitation energies ( $\Delta$ Es) "directly" rather than as differences in two very large numbers. This point of view is what led to the development of so-called equations of motion (EOM) methods within electronic structure theory.

Among the earliest practitioners of EOM methods in the chemistry community were Prof. V. McKoy ${ }^{4}$ and his group at Cal Tech. They imported many ideas and mathematical tools from the nuclear physics literature ${ }^{5}$, where EOM theories had been used to study excited states of nuclei, and the focused their efforts on electronic excitation energies, not IPs or EAs. Because the present Chapter is dedicated to how such methods are used to compute EAs, not much more will be said about the McKoy group's pioneering work on EOM theory for excitation energies, although its ultimate relationship to other excitation-energy methods will be discussed briefly later.

In 1973, the author used the framework of EOM theory ${ }^{6}$ to develop a systematic (i.e., order-by-order in the Møller-Plesset perturbation theory sense) approach for directly computing molecular EAs as eigenvalues of the EOM working equations. It is this development and its subsequent improvement and extensions ${ }^{7}$ by our group and others that we now wish to describe.

\section{Basics of EOM Theory as Applied to EAs}




\section{A. The EA Equations of Motion}

The fundamental working equations of any EOM theory are derived by writing the Schrödinger equations for the neutral and anion states of interest and subtracting the two equations as a first step toward obtaining a single equation that will yield the EA. That is, the EOM theory produces the intensive energy difference directly as an eigenvalue of the working equation. As above, we use $|0, \mathrm{~N}\rangle$ to denote the $0^{\text {th }}$ electronic state of the N-electron neutral and $|\mathrm{K}, \mathrm{N}+1\rangle$ to denote the $\mathrm{K}^{\text {th }}$ state of the $\mathrm{N}+1$-electron anion and write the two Schrödinger equations as

$\mathrm{H}|0, \mathrm{~N}\rangle=\mathrm{E}(0, \mathrm{~N})|0, \mathrm{~N}\rangle$

$\mathrm{H}|\mathrm{K}, \mathrm{N}+1\rangle=\mathrm{E}(\mathrm{K}, \mathrm{N}+1)|\mathrm{K}, \mathrm{N}+1\rangle$.

Because $|0, \mathrm{~N}\rangle$ and $\mid 0, \mathrm{~N}+1>$ contain different numbers of electrons, it is convenient and most common in developing EOM theories of EAs to express the electronic Hamiltonian $\mathrm{H}$ in second-quantized form ${ }^{8}$ :

$H=\Sigma_{i, j} h(i, j) i^{+} j+1 / 2 \Sigma_{i, j, k, 1}<i, j \mid k, 1>i^{=} j^{=} 1 k$

where $h(i, j)$ represents a matrix element of the one-electron operators within the orthonormal molecular spin-orbital basis $\left\{\phi_{j}\right\},<i, j \mid k, 1>$ is a matrix element of the twoelectron operators, and the set of Fermion creation operators $\left\{i^{+}\right\}$create an electron in the $\left\{\phi_{\mathrm{i}}\right\}$ spin-orbitials, whereas the $\{\mathrm{i}\}$ operators destroy such an electron. Writing $\mathrm{H}$ in such a form allows us to use the same $\mathrm{H}$ in Eq. (2a) and Eq. (2b) even those these two Schrödinger equations relate to $\mathrm{N}$ and $\mathrm{N}+1$ electrons, respectively.

The next step in developing an EOM equation is to assume that the anion state $\mid \mathrm{K}, \mathrm{N}+1>$ can be related to the neutral state $\mid 0, \mathrm{~N}>$ through an operator $\mathrm{Q}^{+}(\mathrm{K})$ :

$|\mathrm{K}, \mathrm{N}+1\rangle=\mathrm{Q}^{+}(\mathrm{K}) \mid 0, \mathrm{~N}>$. 
For the EA case at hand, the operator $\mathrm{Q}^{+}(\mathrm{K})$ is usually written in terms of scalar coefficients $t(K, 1)$ multiplied by operators $\mathrm{T}^{+}(1)$ each of which involves adding an electron

$\mathrm{Q}^{+}(\mathrm{K})=\Sigma_{1} \mathrm{t}(\mathrm{K}, 1) \mathrm{T}^{+}(1)$

It has been shown $n^{9}$ that a complete set of such $\mathrm{T}^{+}(1)$ operators consists of the union of sets of operators $\left\{\mathrm{p}^{+}\right\}$that add an electron to a spin-orbital $\phi_{\mathrm{p}}$, operators $\left\{\mathrm{p}^{+} \mathrm{q}^{+} \mathrm{a}\right\}$ that add an electron to $\phi_{\mathrm{p}}$ and excite another electron from $\phi_{\mathrm{a}}$ to $\phi_{\mathrm{q}}$, operators $\left\{\mathrm{p}^{=} \mathrm{q}^{+} \mathrm{r}^{+} \mathrm{ab}\right\}$ that add an electron to $\phi_{\mathrm{p}}$ excite an electron from $\phi_{\mathrm{a}}$ to $\phi_{\mathrm{r}}$ and excite another electron from $\phi_{\mathrm{b}}$ to $\phi_{\mathrm{q}}$ as well as higher-level electron addition and excitation operators up to the highest-level operators that add an electron and induce $\mathrm{N}$ excitations. In labeling these operators, the indices a, b, c, d, etc. are used to denote spin-orbitals occupied in a so-called reference Slater determinant within $\mid 0, N>$ and $p, q, r$, s, etc. are used to denote unoccupied spinorbitals. The reference determinant, which is what defines the concept of occupied and unoccupied spin-orbitals, is usually chosen to be the determinant $\mid 0>$ within

$\left|0, \mathrm{~N}>=\Sigma_{\mathrm{J}=0, \mathrm{M}} \mathrm{C}(0, \mathrm{~J})\right| \mathrm{J}>$

with the largest amplitude $\mathrm{C}(0,0)$, but it has been shown ${ }^{9}$ that $\mid 0>$ can actually be taken to be any determinant within $|0, N\rangle$ that possesses non-zero amplitude.

Using Eq. (4) in Eqs. (2) and subtracting Eq. (2a) from (2b) gives a single equation whose eigenvalue gives the desired EA:

$\left(\mathrm{H} \mathrm{Q}^{+}(\mathrm{K})-\mathrm{Q}^{+}(\mathrm{K}) \mathrm{H}\right)\left|0, \mathrm{~N}>=(\mathrm{E}(\mathrm{K}, \mathrm{N}+1)-\mathrm{E}(0, \mathrm{~N})) \mathrm{Q}^{+}(\mathrm{K})\right| 0, \mathrm{~N}>$

or, in terms of the commutator $\left[\mathrm{H}, \mathrm{Q}^{+}(\mathrm{K})\right]$

$\left[\mathrm{H}, \mathrm{Q}^{+}(\mathrm{K})\right]\left|0, \mathrm{~N}>=\mathrm{E} \mathrm{Q}^{+}(\mathrm{K})\right| 0, \mathrm{~N}>$

where the eigenvalue $\mathrm{E}$ is the negative of the $\mathrm{EA}$. The key point is that one now has a single equation to be solved that produces the intensive EA as its eigenvalue. 
To progress further toward practical implementation, specific choices must be made for how one is going to approximate the neutral-molecule wave function $|0, \mathrm{~N}\rangle$ and at what level one is going to truncate the expansion of the operator $\mathrm{Q}^{+}(\mathrm{K})$ given in Eq. (5). It is also conventional to reduce Eq. (7) to a matrix eigenvalue equation by projecting this equation onto an appropriately chosen space of $\mathrm{N}+1$-electron functions. Let us first deal with the latter issue.

Once the number of $\mathrm{T}^{+}(1)$ operators used to construct $\mathrm{Q}^{+}(\mathrm{K})$ has been chosen (we discuss this choice later), the total number $\mathrm{l}^{\max }$ of $\mathrm{t}(\mathrm{K}, \mathrm{l})$ amplitudes has been determined. Multiplying Eq. (7) on the left by the adjoint $\mathrm{T}(\mathrm{j})$ of any one of the $\mathrm{T}^{+}$operators, and then projecting the resultant equation against $<0, \mathrm{~N} \mid$ gives one form of the working EOM EA equations:

$\Sigma_{1}<0, \mathrm{~N}\left|\mathrm{~T}(\mathrm{j})\left[\mathrm{H}, \mathrm{T}^{+}(\mathrm{l})\right]\right| 0, \mathrm{~N}>\mathrm{t}(\mathrm{K}, \mathrm{l})=\mathrm{E} \Sigma_{1}<0, \mathrm{~N}\left|\mathrm{~T}(\mathrm{j}) \mathrm{T}^{+}(\mathrm{l})\right| 0, \mathrm{~N}>\mathrm{t}(\mathrm{K}, \mathrm{l})$

To make use of this equation, the $\left\langle 0, \mathrm{~N}\left|\mathrm{~T}(\mathrm{j})\left[\mathrm{H}, \mathrm{T}^{+}(\mathrm{l})\right]\right| 0, \mathrm{~N}\right\rangle$ and $\left\langle 0, \mathrm{~N}\left|\mathrm{~T}(\mathrm{j}) \mathrm{T}^{+}(\mathrm{l})\right| 0, \mathrm{~N}\right\rangle$ matrices of dimension $1^{\max } \times 1^{\max }$ must first be evaluated in terms of one-and two-electron integrals (appearing in $\mathrm{H}$ ) and one-, two-, and higher-body density matrices (depending upon the level at which the $\left\{\mathrm{T}^{+}(1)\right\}$ operator expansion is truncated). Subsequently, the EA values (i.e., for the various anion states, $\mathrm{K}$ ) are computed as minus the eigenvalues $\mathrm{E}$ of Eq. (8).

\section{B. The Analogous Equations of Motion for Ionization Potentials}

Before proceeding further, it is useful to explore how this same framework has been used to compute molecular ionization potentials (IPs). It is fairly straightforward to show that an equation analogous to Eq. (7) but reading

$$
<0, \mathrm{~N}\left|\left(\mathrm{H} \mathrm{Q}^{+}(\mathrm{K})-\mathrm{Q}^{+}(\mathrm{K}) \mathrm{H}\right)=(\mathrm{E}(0, \mathrm{~N})-\mathrm{E}(\mathrm{K}, \mathrm{N}-1))<0, \mathrm{~N}\right| \mathrm{Q}^{+}(\mathrm{K})
$$

is valid if the operators $\left\{\mathrm{Q}^{+}(\mathrm{K})\right\}$ are as given in Eq. (5) but with the $\left\{\mathrm{T}^{+}(1)\right\}$ operators defined to include $\left\{a^{+}, a^{+} b^{+} p, a^{+} b^{+} c^{+} q\right.$ r, etc. $\}$. Of course, in Eq. (9), the operators within $\mathrm{Q}^{+}(\mathrm{K})$ act to the left on $<0, \mathrm{~N} \mid$ to generate cationic states. As a result, neutral- 
cation energy differences appear in Eq. (9) and thus this offers a route to computing IPs. Multiplying this equation on the right by any one of the $\mathrm{T}(\mathrm{j})$ operators and then projecting against $|0, N\rangle$ gives

$\Sigma_{1}<0, \mathrm{~N}\left|\left[\mathrm{H}, \mathrm{T}^{+}(\mathrm{l})\right] \mathrm{T}(\mathrm{j})\right| 0, \mathrm{~N}>\mathrm{t}(\mathrm{K}, \mathrm{l})=\mathrm{E} \Sigma_{1}<0, \mathrm{~N}\left|\mathrm{~T}^{+}(\mathrm{l}) \mathrm{T}(\mathrm{j})\right| 0, \mathrm{~N}>\mathrm{t}(\mathrm{K}, \mathrm{l})$

but now the eigenvalues $\mathrm{E}$ denote values of $(\mathrm{E}(0, \mathrm{~N})-\mathrm{E}(\mathrm{K}, \mathrm{N}-1))$, which are the negatives of the IPs.

Thus far, we see that EOMs can be written that allow EAs or IPs to be computed. The fundamental constructs within these equations are as follows:

i. For the EA case, matrix elements $\left.<0, \mathrm{~N}\left|\mathrm{~T}(\mathrm{j})\left[\mathrm{H}, \mathrm{T}^{+}(\mathrm{l})\right]\right| 0, \mathrm{~N}\right\rangle$ involving the commutator of $\mathrm{H}$ with the $\mathrm{T}^{+}(\mathrm{l})$ operators multiplied on the left by a $\mathrm{T}(\mathrm{j})$ operator, as well as an analogous overlap matrix element $<0, \mathrm{~N}\left|\mathrm{~T}(\mathrm{j}) \mathrm{T}^{+}(\mathrm{l})\right| 0, \mathrm{~N}>$;

ii. for the IP case, matrix elements $\left\langle 0, \mathrm{~N}\left|\left[\mathrm{H}, \mathrm{T}^{+}(\mathrm{l})\right] \mathrm{T}(\mathrm{j})\right| 0, \mathrm{~N}\right\rangle$ of the same commutator but with the $T(j)$ operator on the right, as well as the corresponding overlap matrix element $<0, \mathrm{~N}\left|\mathrm{~T}^{+}(\mathrm{l}) \mathrm{T}(\mathrm{j})\right| 0, \mathrm{~N}>$;

iii. the neutral-molecule wave function $|0, N\rangle$ with respect to which the EA or IP is to be evaluated.

\section{The Rank of the Operators}

It is now useful to analyze the density matrix elements ${ }^{10}$ that enter into these equations. Each of the $\mathrm{T}^{+}(\mathrm{j})$ operators contains an odd number of creation or annihilation operators, and the Hamiltonian $\mathrm{H}$ contains two (i.e., $\mathrm{i}^{+} \mathrm{j}$ ) or four (i.e., $\mathrm{i}^{+} \mathrm{j}^{+} \mathrm{lk}$ ) such

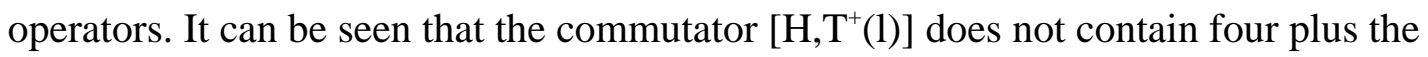
number of creation or annihilation operators as $\mathrm{T}^{+}(1)$, but two fewer. For example, the commutator $\left[\mathrm{i}^{+} \mathrm{j}^{+} \mathrm{lk}, \mathrm{p}^{+} \mathrm{q}^{+} \mathrm{a}\right]$ does not yield any terms with four creation and three annihilation operators but only terms with three creation and two annihilation operators. We say that the act of forming the commutator (which is what causes the higher order operators to cancel) gives rise to a reduction in the rank of the operators. As a result, both the operator products $\mathrm{T}(\mathrm{j})\left[\mathrm{H}, \mathrm{T}^{+}(\mathrm{l})\right]$ and $\left[\mathrm{H}, \mathrm{T}^{+}(\mathrm{l})\right] \mathrm{T}(\mathrm{j})$, which appear in the EA and IP equations of motion, respectively, contain terms only involving both creation and 
annihilation operators equal to the number of creation operators in $\mathrm{T}^{+}(1)$ plus one plus the number of creation operators in $T(j)$. For example, if $T^{+}(1)=p^{+} q^{+} a$ and $T(j)=b^{+} r s$, then $\mathrm{T}(\mathrm{j})\left[\mathrm{H}, \mathrm{T}^{+}(\mathrm{l})\right]$ and $\left[\mathrm{H}, \mathrm{T}^{+}(\mathrm{l})\right] \mathrm{T}(\mathrm{j})$ will contain terms with no more than four creation and four annihilation operators. This means that the density matrices needed to from $<0, \mathrm{~N}\left|\mathrm{~T}(\mathrm{j})\left[\mathrm{H}, \mathrm{T}^{+}(\mathrm{l})\right]\right| 0, \mathrm{~N}>$ and $\left.<0, \mathrm{~N}\left|\left[\mathrm{H}, \mathrm{T}^{+}(\mathrm{l})\right] \mathrm{T}(\mathrm{j})\right| 0, \mathrm{~N}\right\rangle$ will be, at most, fourth order density matrices of the $<0, \mathrm{~N}|\ldots| 0, \mathrm{~N}\rangle$ density.

\section{Equations of Lower Rank for Both EAs and IPs}

Indeed, in the early years of using EOM methods ${ }^{11}$ to compute EAs and IPs, operator manifolds of the form $\left\{\mathrm{T}^{+}(\mathrm{l})\right\}=\left\{\mathrm{p}^{+} ; \mathrm{p}^{+} \mathrm{q}^{+} \mathrm{a}, \mathrm{p}^{+} \mathrm{q}^{+} \mathrm{r}^{+} \mathrm{b}\right.$ a, etc. $\}$ or $\left\{\mathrm{T}^{+}(1)\right\}=\left\{\mathrm{a}^{+}, \mathrm{a}^{+}\right.$ $\mathrm{b}^{+} \mathrm{p}, \mathrm{a}^{+} \mathrm{b}^{+} \mathrm{c}^{+} \mathrm{q}$ r, etc. $\}$ were employed with Møller-Plesset approximations to $\mid 0, \mathrm{~N}>$ (usually taken through first order) to form the kind of matrix elements appearing in Eqs. (8) and (10) and to then evaluate EAs and IPs from their eigenvalues E. However, it became more common to use a combination of the EA and IP EOMs formed by adding Eqs. (8) and (10), while expanding the $\left\{\mathrm{T}^{+}(1)\right\}$ operator manifold to include both $\left\{\mathrm{p}^{+}\right.$;

$\mathrm{p}^{+} \mathrm{q}^{+} \mathrm{a}, \mathrm{p}^{+} \mathrm{q}^{+} \mathrm{r}^{+} \mathrm{b}$ a, etc. $\}$ and $\left\{\mathrm{a}^{+}, \mathrm{a}^{+} \mathrm{b}^{+} \mathrm{p}, \mathrm{a}^{+} \mathrm{b}^{+} \mathrm{c}^{+} \mathrm{q} \mathrm{r}\right.$, etc. $\}$, to simultaneously compute both such energy differences.

To understand why such a combination has proven beneficial, it suffices to examine the form and rank of the operators whose $\langle 0, \mathrm{~N}|\ldots| 0, \mathrm{~N}\rangle$ matrix elements must be evaluated

$\Sigma_{1}<0, \mathrm{~N}\left|\left[\mathrm{H}, \mathrm{T}^{+}(\mathrm{l})\right] \mathrm{T}(\mathrm{j})+\mathrm{T}(\mathrm{j})\left[\mathrm{H}, \mathrm{T}^{+}(\mathrm{l})\right]\right| 0, \mathrm{~N}>\mathrm{t}(\mathrm{K}, \mathrm{l})$

$=\mathrm{E} \Sigma_{1}<0, \mathrm{~N}\left|\mathrm{~T}^{+}(\mathrm{l}) \mathrm{T}(\mathrm{j})+\mathrm{T}(\mathrm{j}) \mathrm{T}^{+}(\mathrm{l})\right| 0, \mathrm{~N}>\mathrm{t}(\mathrm{K}, \mathrm{l})$

Recall that the $\mathrm{T}^{+}(\mathrm{j})$ operators contain an odd number of creation or annihilation operators. Each of the products $\left[H, \mathrm{~T}^{+}(\mathrm{l})\right] \mathrm{T}(\mathrm{j}), \mathrm{T}(\mathrm{j})\left[\mathrm{H}, \mathrm{T}^{+}(\mathrm{l})\right], \mathrm{T}^{+}(\mathrm{l}) \mathrm{T}(\mathrm{j})$, and $\mathrm{T}(\mathrm{j}) \mathrm{T}^{+}(\mathrm{l})$ thus contain an even number of such operators. However, because of the fundamental anti-commutation properties of these operators

$i^{+} j+j i^{+}=\delta_{i, j}$ 
$i j+j i=0$

$\mathrm{i}^{+} \mathrm{j}^{+}+\mathrm{j}^{+} \mathrm{i}^{+}=0$

it can easily be shown that the operator combinations $\mathrm{T}^{+}(\mathrm{l}) \mathrm{T}(\mathrm{j})+\mathrm{T}(\mathrm{j}) \mathrm{T}^{+}(\mathrm{l})$ and $\left[\mathrm{H}, \mathrm{T}^{+}(\mathrm{l})\right]$ $\mathrm{T}(\mathrm{j})+\mathrm{T}(\mathrm{j})\left[\mathrm{H}, \mathrm{T}^{+}(\mathrm{l})\right]$ contain one fewer creation and one fewer annihilation operator than does either of the two terms in the sums. So, by combining the EA and IP EOMs, one effects a rank reduction in the operators appearing in the equations although the dimensions of the matrices one needs to construct are doubled (because the $\left\{\mathrm{T}^{+}(1)\right\}$ operator manifold was doubled. The rank reduction is important because it means that the density matrices that need to be evaluated to compute the $\langle 0, N|\ldots| 0, N\rangle$ matrix elements are of lower rank in Eq. (11) than in either Eq. (8) or Eq. (10). As we said, it has become more common to use the combined EA and IP Eq. (11) because lower-order density matrices are required.

\section{E. Summary}

Thus far, we have seen how one can obtain eigenvalue equations in which the energy eigenvalues correspond to the intensive EAs (or IPs) by postulating that the anion (or cation) wave function can be related to the neutral-molecule wave function through an operator. We have also seen how the EA and IP equations of motion can be combined to generate a combined EOM from which both EAs and IPs can be obtained. The advantage to the latter approach is that the operators appearing in the resultant equations are of lower rank and thus lower-order density matrices must be evaluated to carry out the calculations. Let us now move on to address more specific embodiments of such EOM theories that result from different choices of the neutral-molecule wave function and of the operator connecting the neutral and anion wave functions.

\section{Practical Implementations of EOM Theories for EAs}


The basic ideas underlying any EOM method for computing EAs or IPs appear above. However, in any specific embodiment of such a method, one must commit to i. a specific approximation to the neutral-molecule wave function $|0, \mathrm{~N}\rangle$, ii. a specific choice of how large an operator manifold $\left\{\mathrm{T}^{+}(1)\right\}$ to employ, and iii. how to solve the resultant EOM equations for the eigenvalues $\mathrm{E}$ that then produce the EAs or IPs. In the following subsections, we describe the most commonly used choices for these three issues.

\section{A. The Møller-Plesset Based Approximations}

In the earliest implementation of EOM approaches to EAs, the author's group ${ }^{6,11}$ chose to represent the $|0, N\rangle$ wave function in a Møller-Plesset (MP) expansion

$\mid 0, N>=\psi^{0}+\psi^{1}+\psi^{2}+\ldots$

with the single-determinant unrestricted Hartree-Fock (HF) function being $\psi^{0}$ and the corresponding neutral-molecule HF Hamiltonian being $\mathrm{H}^{0}$. The operator manifold $\left\{\mathrm{T}^{+}(1)\right\}$ was taken to consist of $\left\{\mathrm{p}^{+} ; \mathrm{p}^{+} \mathrm{q}^{+} \mathrm{a}\right\}$ and $\left\{\mathrm{a}^{+}, \mathrm{a}^{+} \mathrm{b}^{+} \mathrm{p}\right\}$. This choice of operator manifold was shown to be capable of producing EAs and IPs that were precise through third order ${ }^{12}$ in the MP perturbation.

The resultant variant of Eq. (11) was not solved by finding the eigenvalues of this equation whose dimension is the sum of the dimensions of the $\left\{\mathrm{p}^{+} ; \mathrm{p}^{+} \mathrm{q}^{+} \mathrm{a}\right\}$ and $\left\{\mathrm{a}^{+}, \mathrm{a}^{+} \mathrm{b}^{+}\right.$ p) operator manifolds. Rather, that large matrix eigenvalue problem was partitioned ${ }^{6}$ using subspaces defined by the $\left\{\mathrm{p}^{+}, \mathrm{a}^{+}\right\}$operators as the primary subspace and the $\left\{\mathrm{p}^{+} \mathrm{q}^{+}\right.$ $\mathrm{a}, \mathrm{a}^{+} \mathrm{b}^{+} \mathrm{p}$ \} operators as the secondary subspace. The partitioned eigenvalue problem

$\sum_{\mathrm{j}=\mathrm{a}, \mathrm{p}} \mathrm{H}_{\mathrm{i}, \mathrm{j}}(\mathrm{E}) \mathrm{X}_{\mathrm{j}}=\mathrm{E} \mathrm{X}_{\mathrm{i}}$

whose dimension was that of the $\left\{\mathrm{p}^{+}, \mathrm{a}^{+}\right\}$operator space was used to find the eigenvalues E. When the elements of the partitioned matrices were evaluated through second order in the MP series, the following expression was obtained for the matrix elements $\mathrm{H}_{\mathrm{i}, \mathrm{j}}$; 
$\mathrm{H}_{\mathrm{i}, \mathrm{j}}(\mathrm{E})=\varepsilon_{\mathrm{i}} \delta_{\mathrm{i}, \mathrm{j}}-\Sigma_{\mathrm{p}, \mathrm{q}, \mathrm{a}}<\mathrm{i}, \mathrm{a} \mid \mathrm{p}, \mathrm{q}>\langle\mathrm{p}, \mathrm{q}| \mathrm{j}, \mathrm{a}>/\left(\varepsilon_{\mathrm{p}}+\varepsilon_{\mathrm{q}}-\varepsilon_{\mathrm{a}}-\mathrm{E}\right)$

$+\sum_{\mathrm{a}, \mathrm{b}, \mathrm{p}}<\mathrm{i}, \mathrm{p} \mid \mathrm{a}, \mathrm{b}>\langle\mathrm{a}, \mathrm{b}| \mathrm{j}, \mathrm{p}>/\left(\varepsilon_{\mathrm{a}}+\varepsilon_{\mathrm{b}}-\varepsilon_{\mathrm{p}}-\mathrm{E}\right)$

The expression for $\mathrm{H}_{\mathrm{i}, \mathrm{j}}(\mathrm{E})$ valid through third order in the MP series is more complicated and is given in Eqs. (31)-(37) of ref. 6, which are reproduced for historical perspective in Fig. 1. 


$$
\begin{aligned}
& S_{n \times m, q \delta p}=\delta_{m q} \delta_{\alpha p} \delta_{m p}, \\
& S_{s p \gamma, \alpha<\beta}=\delta_{t \Delta \alpha} \delta_{p q} \delta_{\tau \beta,} \\
& S_{t_{p \gamma} \text { ingm }}=0 \text {, } \\
& A_{i j}=\hbar_{i j} \epsilon_{i}+\sum_{k_{1}, f}\left\langle i k_{i} ; j\right\rangle F_{i j,}
\end{aligned}
$$

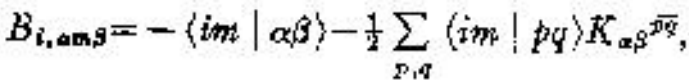

$$
\begin{aligned}
& +\sum_{\gamma, p}\left[\langle i \gamma \mid p \alpha\rangle K_{\overline{d \gamma}} \overline{m p}^{\bar{m}}-\langle i \gamma \mid p \beta\rangle K_{\overline{n \gamma}}^{\overline{m p}}\right] \text {, } \\
& B_{i, \text { nam }}=\langle i \alpha \mid m n\rangle+\frac{1}{2} \sum_{>4}\langle i \alpha \mid \delta \gamma\rangle K_{\overline{F_{\gamma}}}^{\mathrm{twn}}
\end{aligned}
$$

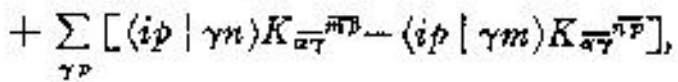

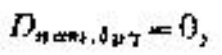

$$
\begin{aligned}
& D_{n a+m, q \delta \mu}=\delta_{n q} \delta_{\alpha g} \delta_{m, p} E_{\alpha}^{n n} \text {, }
\end{aligned}
$$

and

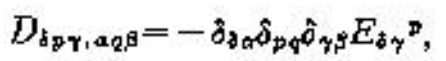

where

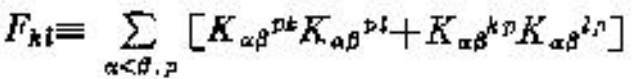

$$
\begin{aligned}
& -\sum_{x<q, a}\left[K_{a 1^{p q q}} K_{a b}^{p q}+K_{i \alpha}^{p q} K_{k \alpha a}^{p q}\right]
\end{aligned}
$$

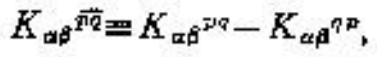

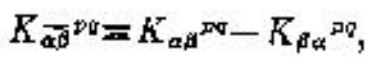

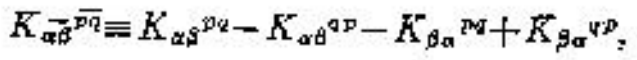

$$
\begin{aligned}
& E_{\mathrm{a}}^{\mathrm{mnn}}=\varepsilon_{\mathrm{n}}+\epsilon_{\mathrm{n}}-\epsilon_{\mathrm{a}}-\langle\alpha m \mid \alpha m\rangle-\langle\alpha n \mid \alpha n\rangle+\langle m n \mid m n\rangle,
\end{aligned}
$$

$$
\mathrm{H}\left(\Delta E_{\mathrm{\lambda}}{ }^{N}\right) \mathrm{X}(\lambda)=\Delta E_{\mathrm{\lambda}}{ }^{N} \mathrm{X}(\lambda),
$$

where the matrix elements of $H_{i j}\left(\Delta E_{2}{ }^{N}\right)$ are compactly written, in terms of Hartree-Fock information only, as

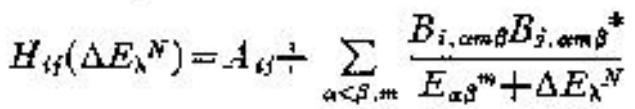

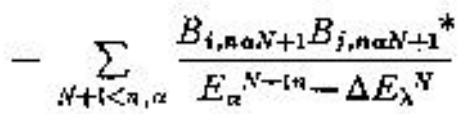

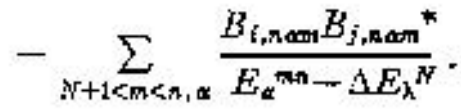

Figure 1. Working equations of third-order (in Møller-Plesset perturbation theory) equations of motion of ref. 6 . 
These third-order equations have been used in many applications in which molecular EAs have been computed for a wide variety of species as illustrated in refs. 11. Clearly, all of the quantities needed to form the second- or third- order EOM matrix elements $\mathrm{H}_{\mathrm{j}, \mathrm{k}}$ are ultimately expressed in terms of the orbital energies $\left\{\varepsilon_{k}\right\}$ and two-electron integrals $<\mathrm{j}, \mathrm{k} \mid \mathrm{l}, \mathrm{h}>$ evaluated in the basis of the Hartree-Fock orbitals that form the starting point of the Møller-Plesset theory. However, as with most electronic structure theories, much effort has been devoted to recasting the working EOM equations in a manner that involves the atomic-orbital two-electron integrals rather than the molecular-orbital based integrals. Because such technical matters are beyond the scope of this work, we will not delve into them further.

\section{B. Relationship to Greens Functions/Propagators}

It turns out that in the early 1970s, several groups had taken a different approach to the evaluation of atomic and molecular electronic energy differences using what were called Greens functions (GF) or propagators. Linderberg and Öhrn pioneered ${ }^{13}$ the use of such methods in quantum chemistry, while Cederbaum and co-workers ${ }^{14}$, Reinhardt and Doll ${ }^{15}$, Taylor, Yaris, and co-workers ${ }^{16}$ and Pickup and Goscinski ${ }^{17}$ were among the first to apply the methods using an ab initio approach. Purvis and Öhrn ${ }^{18}$ soon thereafter expanded the range of the theory to include open-shell systems.

These theories were derived from consideration of the following time-dependent matrix elements:

$$
\begin{aligned}
& \left.\mathrm{G}_{\mathrm{j}, \mathrm{k}}(\mathrm{t})=(1 / \mathrm{i} \hbar) \Theta(\mathrm{t})<0, \mathrm{~N}\left|\exp (\mathrm{iH} t / \hbar) \mathrm{j}^{+} \exp (-\mathrm{iH} \mathrm{t} / \hbar) \mathrm{k}\right| 0, \mathrm{~N}\right\rangle \\
& \left.+(1 / \mathrm{i} \hbar) \Theta(-\mathrm{t})<0, \mathrm{~N}\left|\mathrm{k} \exp (\mathrm{iH} t / \hbar) \mathrm{j}^{+} \exp (-\mathrm{iH} \mathrm{t} / \hbar)\right| 0, \mathrm{~N}\right\rangle
\end{aligned}
$$

Here, $\Theta(t)$ is the Heaviside step function, which equals unity when $t$ is positive and zero when $\mathrm{t}$ is negative, $\mathrm{j}^{+}$and $\mathrm{k}$ are the same creation and annihilation operators discussed earlier, and $|0, N\rangle$ is the neutral-molecule reference wave function. Introducing complete sets of $\mathrm{N}-1$ and $\mathrm{N}+1$ electron Hamiltonian eigenfunctions into the first and second terms 
in Eq. (16), one observes time dependences varying as $\exp (\mathrm{i}[\mathrm{E}(0, \mathrm{~N})-\mathrm{E}(\mathrm{K}, \mathrm{N}-1)] \mathrm{t} / \hbar)$ and $\exp (\mathrm{i}[\mathrm{E}(\mathrm{K}, \mathrm{N}+1)-\mathrm{E}(0, \mathrm{~N})] \mathrm{t} / \hbar)$, respectively.

Taking the time derivative of Eq. (16), one obtains expressions involving commutators of the form $\left[\mathrm{H}, \mathrm{j}^{+}\right] \mathrm{k}$ and $\mathrm{k}\left[\mathrm{H}, \mathrm{j}^{+}\right]$just as one finds in Eq. (11). By analyzing the resulting time-derivative equations, workers in this field were able to obtain equations that such $\mathrm{G}_{\mathrm{j}, \mathrm{k}}(\mathrm{t})$ matrix elements obey (n.b., these were called the equations of motion for these quantities). The workers named above were able to express the resulting equations in terms of one-and two-electron integrals and corresponding density matrices much as the author had done within the EOM framework. In fact, it turns out that the final working equations of the so-called one-electron Greens function or electron propagator defined in Eq. (16), when Fourier transformed from the time to the energy domain, were exactly the same as the EOM equations given above (i.e., Eq. (15) and those shown in Fig. 1).

Especially in recent years, much of the work aimed at calculating EAs and IPs using these direct-calculation EOM and GF methods has been performed within the notation of Greens functions and has been carried out by Vince Ortiz's group ${ }^{19}$ as well as by the Cederbaum group. To further illustrate the impact that such advances have had within the quantum chemistry community, we note that the Ortiz group has implemented various (i.e., Møller-Plesset and other) variants of these theories within the highly successful Gaussian ${ }^{20}$ suite of computer codes as a result of which many workers worldwide now employ EOM-type methods to evaluate EAs and IPs.

\section{The Natural Orbital or Extended Koopmans' Theorem Approach}

In the mid 1970s, R. G. Parr and co-workers ${ }^{21}$ and, independently, D. Smith and co-workers ${ }^{22}$ proposed to use an equation such as Eq.(10) for computing IPs and they referred to these methods as natural orbital or extended Koopmans' theorem theories. Subsequently, E. Andersen and the author analyzed ${ }^{23}$ the working equations of this approach through second and third order in the MP series and noted differences between them and the Greens function and equivalent EOM theories computed through these same orders. Of course, based on the discussion of Sec. II. D, these differences relate to the ranks of the operators appearing in the working equations and are not surprising. More 
recently, Cioslowski and co-workers ${ }^{24}$ have shown that these extended Koopmans' theorem approaches indeed offer a very efficient and reasonably accurate route to computing IPs or EAs, so it is likely that these methods will continue to develop.

\section{Multi-configuration Based Approximations}

Following on the proof by R. Manne that the operator spaces $\left\{\mathrm{T}^{+}(1)\right\}=\left\{\mathrm{p}^{+} ; \mathrm{p}^{+} \mathrm{q}^{+} \mathrm{a}\right.$, $\mathrm{p}^{+} \mathrm{q}^{+} \mathrm{r}^{+} \mathrm{b}$ a, etc. $\}$ and $\left\{\mathrm{T}^{+}(\mathrm{l})\right\}=\left\{\mathrm{a}^{+}, \mathrm{a}^{+} \mathrm{b}^{+} \mathrm{p}, \mathrm{a}^{+} \mathrm{b}^{+} \mathrm{c}^{+} \mathrm{q} \mathrm{r}\right.$, etc. $\}$ can be used (i.e., is capable of forming complete sets of ion states) even when no single determinant forms a dominant component of the neutral-molecule wave function $|0, N\rangle$, the author's group extended the combined EA and IP EOM theory to the case in which $|0, N\rangle$ is of an arbitrary multi-configuration self-consistent field (MC-SCF) form ${ }^{25}$ and the ionization operator manifold $\left\{\mathrm{T}^{+}(1)\right\}$ included operators of the form $\left\{\mathrm{p}^{+} ; \mathrm{p}^{+} \mathrm{q}^{+} \mathrm{a}\right\}$ and $\left\{\mathrm{a}^{+}, \mathrm{a}^{+} \mathrm{b}^{+} \mathrm{p}\right\}$. The resultant working equations were written as in Eq. (14), with the $\mathrm{H}_{\mathrm{j}, \mathrm{k}}$ matrix elements given in Eqs. (18) of ref. 25, which we do not reproduce here because of their complexity. The primary additional difficulty involved in implementing these multiconfiguration-based equations is the fact that three-electron density matrices $<0, \mathrm{~N} \mid \mathrm{i}^{+} \mathrm{j}^{+} \mathrm{k}^{+}$ $1 \mathrm{~h} \mathrm{n}|0, \mathrm{~N}\rangle$ taken with respect to the MC-SCF wave function $|0, \mathrm{~N}\rangle$ are involved. These density matrices arise when the commutators $\left[\mathrm{H}, \mathrm{p}^{+} \mathrm{q}^{+} \mathrm{a}\right]$ and $\left[\mathrm{H}, \mathrm{a}^{+} \mathrm{b}^{+} \mathrm{p}\right]$ are evaluated.

\section{E. Coupled-Cluster Based EOM}

The use of coupled-cluster (CC) wave functions within EOM theory for excitation energies, IPs, and EAs has been developed ${ }^{26,27}$ upon slightly different lines than outlined in Sec. II. The CC wave function ansatz for $|0, N\rangle$ is written as usual in terms of an exponential operator acting on a single-determinant (e.g., unrestricted HF) "reference function" $\mid 0>$

$|0, \mathrm{~N}>=\exp (\mathrm{T})| 0>$

The so-called cluster operator $\mathrm{T}$ is expressed in terms of spin-orbital excitation operators of the form $\left\{\mathrm{T}_{1}\right\}=\left\{\mathrm{p}^{+} \mathrm{a}\right\},\left\{\mathrm{T}_{2}\right\}=\left\{\mathrm{p}^{+} \mathrm{q}^{+} \mathrm{b} \mathrm{a}\right\},\left\{\mathrm{T}_{4}\right\}=\left\{\mathrm{p}^{+} \mathrm{q}^{+} \mathrm{r}^{+} \mathrm{c} \mathrm{b}\right.$ a $\}$, etc. with $\mathrm{T}_{\mathrm{k}}$ relating 
to the excitation of k electrons from occupied spin-orbitals (a, b, c. etc.) to virtual spinorbitals (p, q, r, etc.). Prior to forming any EA EOM, the neutral-molecule CC equations need to be solved for the amplitudes $\left\{t_{n}\right\}$ that multiply the $\left\{T_{n}\right\}$ operators to form the CC $\mathrm{T}$ operator. For completeness, recall that the $\mathrm{CC}$ equations are formed by manipulating the Schrödinger equation

$\mathrm{H} \exp (\mathrm{T})|0>=\mathrm{E} \exp (\mathrm{T})| 0>$

to read

$\exp (-\mathrm{T}) \mathrm{H} \exp (\mathrm{T})|0>=\mathrm{E}| 0>$

and subsequently projecting this equation against functions $\left\{<0 \mid \mathrm{T}_{\mathrm{n}}^{+}\right\}$. Because the $\mathrm{T}$ operator contains only creation operators for unoccupied spin-orbitals and annihilation operators for occupied spin-orbitals, it turns out that the commutator expansion

$\exp (-\mathrm{T}) \mathrm{H} \exp (\mathrm{T})=\mathrm{H}-[\mathrm{T}, \mathrm{H}]+1 / 2[\mathrm{~T},[\mathrm{~T}, \mathrm{H}]]-1 / 3 ![\mathrm{T},[\mathrm{T},[\mathrm{T}, \mathrm{H}]]]$

$+1 / 4 ![\mathrm{T},[\mathrm{T},[\mathrm{T},[\mathrm{T}, \mathrm{H}]]]]$

exactly truncates at the fourth order term. So, the final working equations of $\mathrm{CC}$ theory can be written as

$<0 \mid \mathrm{T}_{\mathrm{n}}^{+}\{\mathrm{H}-[\mathrm{T}, \mathrm{H}]+1 / 2[\mathrm{~T},[\mathrm{~T}, \mathrm{H}]]-1 / 3 ![\mathrm{T},[\mathrm{T},[\mathrm{T}, \mathrm{H}]]]$

$+1 / 4 ![\mathrm{T},[\mathrm{T},[\mathrm{T},[\mathrm{T}, \mathrm{H}]]]]\} \mid 0>=0$.

Once the CC amplitides $\left\{\mathrm{t}_{\mathrm{n}}\right\}$ are determined by solving these quartic equations, the CC energy is computed as

$<0 \mid \mathrm{H}-[\mathrm{T}, \mathrm{H}]+1 / 2[\mathrm{~T},[\mathrm{~T}, \mathrm{H}]]-1 / 3 ![\mathrm{T},[\mathrm{T},[\mathrm{T}, \mathrm{H}]]]$ 
$+1 / 4$ ! $[\mathrm{T},[\mathrm{T},[\mathrm{T},[\mathrm{T}, \mathrm{H}]]]] \mid 0>=\mathrm{E}$.

The operator $\mathrm{Q}^{+}(\mathrm{K})$ that maps $\mid 0, \mathrm{~N}>$ into an anion or cation state is expressed as in Eq. (5) with the $\left\{\mathrm{T}^{+}(1)\right\}$ operators including $\left\{\mathrm{T}^{+}(1)\right\}=\left\{\mathrm{p}^{+} ; \mathrm{p}^{+} \mathrm{q}^{+} \mathrm{a}, \mathrm{p}^{+} \mathrm{q}^{+} \mathrm{r}^{+} \mathrm{b}\right.$ a, etc. $\}$ when EAs are to be computed. The basic EOM analogous to Eq. (7b) is then written as

$\left[\mathrm{H}, \mathrm{Q}^{+}(\mathrm{K})\right] \exp (\mathrm{T})\left|0>=\mathrm{E} \mathrm{Q}^{+}(\mathrm{K}) \exp (\mathrm{T})\right| 0>$.

Multiplying on the left by $\exp (-\mathrm{T})$ and realizing that $\mathrm{T}$ and $\mathrm{Q}^{+}(\mathrm{K})$ commute reduces this equation to

$\left[\mathrm{H}^{\prime}, \mathrm{Q}^{+}(\mathrm{K})\right] \mid 0>=\mathrm{E} \mathrm{\textrm {Q } ^ { + } ( \mathrm { K } ) | 0 >}$

where

$H^{\prime}=\exp (-T) H \exp (T)$

which can be expanded as in Eq. (20) to involve at most quartic terms in the $\left\{\mathrm{t}_{\mathrm{n}}\right\}$ amplitudes. Then, multiplying on the left by $<0 \mid \mathrm{T}(\mathrm{j})$ reduces the EOM equations to their final working form

$\Sigma_{1}<0 \mid \mathrm{T}(\mathrm{j})[\{\mathrm{H}-[\mathrm{T}, \mathrm{H}]+1 / 2[\mathrm{~T},[\mathrm{~T}, \mathrm{H}]]-1 / 3 ![\mathrm{T},[\mathrm{T},[\mathrm{T}, \mathrm{H}]]]$

$+1 / 4$ ! [T, $[\mathrm{T},[\mathrm{T},[\mathrm{T}, \mathrm{H}]]]]\}, \mathrm{T}^{+}(\mathrm{l})\left|0>\mathrm{t}(\mathrm{K}, \mathrm{l})=\mathrm{E} \Sigma_{1}<0\right| \mathrm{T}(\mathrm{j}) \mathrm{T}^{+}(\mathrm{l}) \mid 0>\mathrm{t}(\mathrm{K}, \mathrm{l})$.

This set of matrix eigenvalue equations are then solved to obtain $\mathrm{E}$ which gives the EA. Such so-called electron-attached equations of motion (EA-EOM) approaches have proven highly successful ${ }^{27}$ in computing EAs of a wide range of atoms and molecules primarily because the coupled-cluster treatment of electron correlation provides such a highly accurate treatment of the dynamical electron correlation. 


\section{Some Tricks of the Trade}

\section{A. Calculating EAs as IPs}

In this discussion, we have focused on computing EAs by forming a neutralmolecule wave function $|0, N\rangle$ and computing the EA as an eigenvalue of an EOM. Consider applying such an approach to evaluate the EA of the $X^{2} \Pi$ state of the NO molecule. Because the X-state wave function of NO is spatially degenerate (i.e., the $\pi_{\mathrm{x}}$ and $\pi_{\mathrm{y}}$ orbitals should be degenerate), one may encounter artifactual symmetry breaking when forming this neutral-molecule function. That is, the $\pi_{x}$ and $\pi_{y}$ orbitals may not turn out to be degenerate. It would then be unwise to use this symmetry-broken wave function to compute any property of this state of NO, including the EA. To overcome such difficulties, one could use the $\mathrm{X}^{3} \Sigma^{+}$state of $\mathrm{NO}^{-}$as $|0, \mathrm{~N}\rangle$ and employ an EOM method to evaluate the IP of $\mathrm{NO}^{-}$(actually the electron detachment energy of NO). The advantage to this approach is that the open-shell ${ }^{3} \Sigma^{+}$state of $\mathrm{NO}^{-}$would not be susceptible to symmetry breaking because it is not spatially degenerate. This example shows that it may sometimes be better to compute an EA of a molecule as the IP of the corresponding anion.

\section{B. Treating Metastable Anion States}

A different kind of problem arises when one attempts to compute the EA of a molecule whose anion is not electronically bound relative to the corresponding neutral. For example, the $\mathrm{X}^{1} \Sigma_{\mathrm{g}}{ }^{+}$state of the $\mathrm{N}_{2}$ molecule does not bind an electron to form an electronically stable anion. Instead the $\mathrm{X}^{2} \Pi_{\mathrm{g}}$ state of $\mathrm{N}_{2}^{-}$, formed by adding an electron to the $\pi_{\mathrm{g}}$ anti-bonding orbital of $\mathrm{N}_{2}$ is a so-called resonance state that lies higher in energy than $\mathrm{N}_{2}$ and can spontaneously eject its excess electron. One cannot simply employ conventional basis sets and ab initio electronic structure methods (including EOM methods) to correctly determine the energies of such states.

The most common and powerful tool for studying such metastable states theoretically is the stabilization method (SM). This method, pioneered by Professor Howard Taylor's group ${ }^{28}$, involves embedding the system of interest (e.g., the $\mathrm{N}_{2}{ }^{-1}$ anion) 
within a finite "box" in order to convert the continuum of states corresponding, for example, to $\mathrm{N}_{2}+\mathrm{e}$, into discrete states that can be handled by, for example, the EOM method. By then varying the size of the box, one can vary the energies of the discrete states that correspond to $\mathrm{N}_{2}+\mathrm{e}^{-}$(i.e., one varies the box size to vary the kinetic energy $\mathrm{KE}$ of the orbital containing the excess electron). As the box size is varied, one eventually notices (e.g., by plotting the orbitals) that one of the $\mathrm{N}_{2}+\mathrm{e}^{-}$states obtained in the EOM process possesses a significant amount of valence character. That is, one such state has significant amplitude not only at large-r but also in the region of the two nitrogen centers. It is this state that corresponds to the metastable resonance state, and it is the EOM eigenvalue $\mathrm{E}$ of this state that provides the stabilization estimate of the resonance state energy relative to that of the neutral $\mathrm{N}_{2}$.

Let us continue using $\mathrm{N}_{2}^{-1}$ as an example for how one usually varies the box within which the anion is constrained. One uses a conventional atomic orbital basis set that likely includes $\mathrm{s}$ and $\mathrm{p}$ functions on each $\mathrm{N}$ atom, perhaps some polarization $\mathrm{d}$ functions and some conventional diffuse $\mathrm{s}$ and $\mathrm{p}$ orbitals on each $\mathrm{N}$ atom. These basis orbitals serve primarily to describe the motions of the electrons within the usual valence regions of space. To this basis, one appends an extra set of diffuse $\pi$-symmetry orbitals. These orbitals could be $p_{\pi}$ (and maybe $d_{\pi}$ ) functions centered on each nitrogen atom, or they could be $\mathrm{d}_{\pi}$ obitals centered at the midpoint of the N-N bond. Either choice can be used because one only needs a basis capable of describing the large-r $\mathrm{L}=2$ character of the metastable ${ }^{2} \Pi_{g}$ state's wave function. One usually would not add just one such function; rather several such functions, each with an orbital exponent $\alpha_{\mathrm{J}}$ that characterizes its radial extent, would be used. Let us assume, for example, that $\mathrm{K}$ such additional diffuse $\pi$ functions have been used.

Next, using the conventional atomic orbital basis as well as the K extra $\pi$ basis functions, one carries out an EOM calculation for the EA of the $\mathrm{N}_{2}^{-1}$ anion. In this calculation, one tabulates the energies of many (say M) of the EOM EA eigenvalues. One then scales the orbital exponents $\left\{\alpha_{J}\right\}$ of the K extra $\pi$ basis orbitals by a factor $\eta$ : $\alpha_{J} \rightarrow \eta$ $\alpha_{\mathrm{J}}$ and repeats the calculation of the energies of the M lowest EOM eigenvalues. This scaling causes the extra $\pi$ basis orbitals to contract radially (if $\eta>1$ ) or to expand radially (if $\eta<1$ ). It is this basis orbital expansion and contraction that produces 
expansion and contraction of the "box" discussed above. That is, one does not employ a box directly; instead, one varies the radial extent of the more diffuse basis orbitals to simulate the box variation.

If the conventional orbital basis is adequate, one finds that the extra $\pi$ orbitals, whose exponents are being scaled, do not affect appreciably the energy of the neutral $\mathrm{N}_{2}$ system. This can be probed by plotting the $\mathrm{N}_{2}$ energy (computed as $\langle 0, \mathrm{~N}|\mathrm{H}| 0, \mathrm{~N}\rangle$ ) as a function of the scaling parameter $\eta$; if the energy varies little with $\eta$, the conventional basis is adequate.

In contrast to plots of the neutral $\mathrm{N}_{2}$ energy vs. $\eta$, plots of the energies of the $\mathrm{M}$ $\mathrm{N}_{2}^{-1}$ anion states relative to the energy of $\mathrm{N}_{2}$, obtained as EOM eigenvalues, show significant $\eta$-dependence as Fig. 2 illustrates.

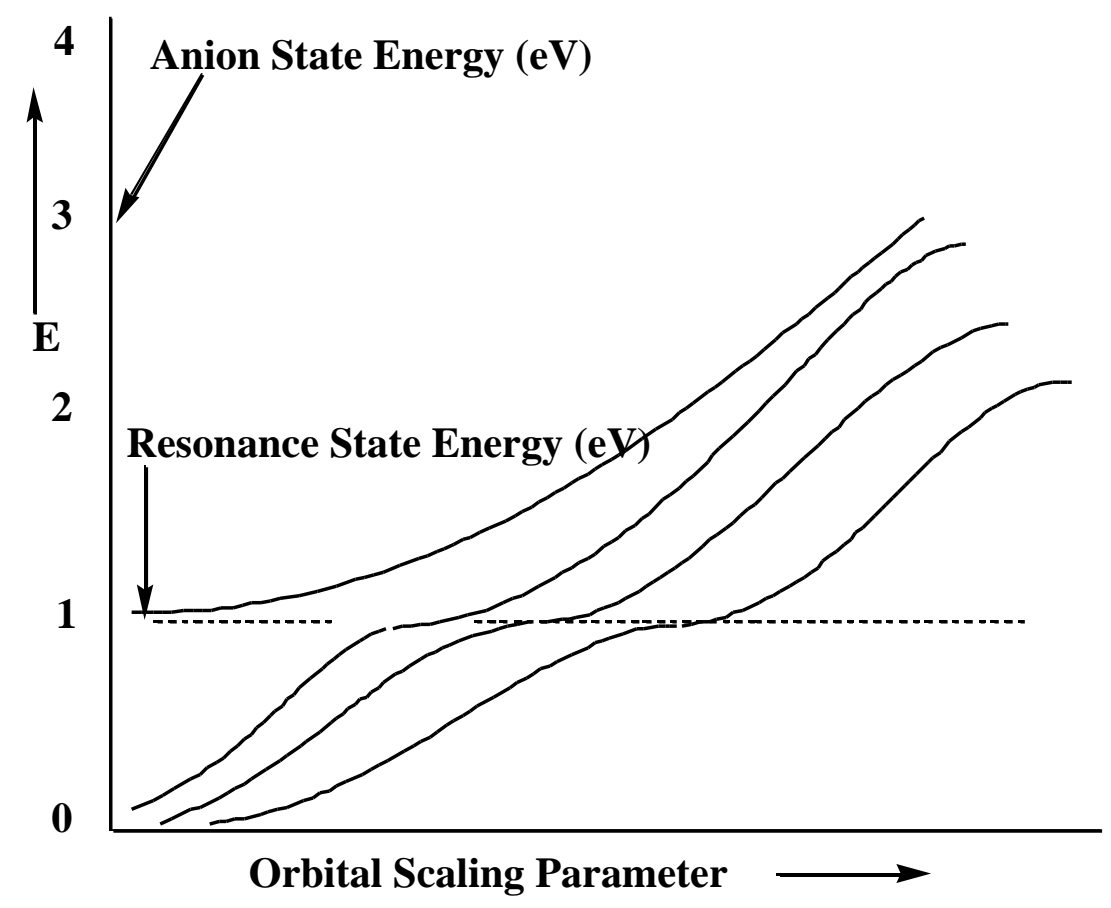

Figure 2. Plots of the EOM EA eigenvalues for several anion states vs. the orbital scaling parameter $\eta$. Note the avoided crossing of state energies near $1 \mathrm{eV}$. 
What does such a stabilization plot tell us and what do the various branches of the plot mean? First, we notice that each of the plots of the energy of an anion state (relative to the neutral molecule's energy, which is independent of $\eta$ ) grows with increasing $\eta$. This $\eta$-dependence arises from the $\eta$-scaling of the extra diffuse $\pi$ basis orbitals. Because most of the amplitude of such basis orbitals lies outside the valence region, the kinetic energy is the dominant contributor to such states' relative energies. Because $\eta$ enters into each orbital as $\exp \left(-\eta \alpha r^{2}\right)$, and because the kinetic energy operator involves the second derivative with respect to $r$, the kinetic energies of orbitals dominated by the diffuse $\pi$ basis functions vary as $\eta^{2}$. It is this quadratic growth with $\eta$ that is shown in Fig 2.

For small $\eta$, all of the $\pi$ diffuse basis functions have their amplitudes concentrated at large $r$ and have low kinetic energy. As $\eta$ grows, these functions become more radially compact and their kinetic energies grow. For example, note the three lowest energies shown above in Fig. 2 increasing from near zero as $\eta$ grows. As $\eta$ further increases, one reaches a point at which the third and fourth anion-state energies in Fig. 2 undergo an avoided crossing. At this $\eta$ value, if one examines the nature of the two anion wave functions (obtained as in Eq.(4)) whose energies avoid one another, one finds they contain substantial amounts of both valence and extra diffuse $\pi$ function character. Just to the left of the avoided crossing, the lower-energy state (the third state in Fig. 2 for small $\eta$ ) contains predominantly extra diffuse $\pi$ orbital character, while the higher-energy state (the fourth state) contains largely valence $\pi^{*}$ orbital character. To the right of the avoided crossing, the situation is reversed- the lower-energy state (the third state in Fig. 2 for small $\eta$ ) contains predominantly valence orbital character, while the higher-energy state (the fourth state) contains largely diffuse orbital character

However, at the special value of $\eta$ where these two states nearly cross, the kinetic energy of the diffuse state (as well as its radial size and de Broglie wavelength) are appropriate to connect properly with the valence state to form a single resonance state. By connect properly we mean that the two states have wave function amplitudes, phases, and slopes that match. It is such boundary condition matching of valence-range and longrange character in the wave function that the stabilzation method achieves. So, at this special $\eta$ value, one can achieve a description of the resonance state that correctly describes this state both in the valence region and in the large-r region. Only by tuning 
the energy of the large-r states using the $\eta$ scaling can one obtain this proper boundary condition matching.

If one attempts to study metastable anion states without carrying out such a stabilization study, one is doomed to failure, even if one employs an extremely large and flexible set of diffuse basis functions. In such a calculation, one will certainly obtain a large number of anion "states" with energies lying above that of the neutral, but one will not be able to select from these states the one that is the true resonance state because the true state will be buried in the myriad of "states".

In summary, by carrying out a series of anion-state energy calculations for several states and plotting them vs. $\eta$, one obtains a stabilization graph. By examining this graph and looking for avoided crossings, one can identify the energies at which metastable resonances occur. It is absolutely critical to identify these resonance energies if one wishes to probe metastable anions. It is also possible ${ }^{29}$ to use the shapes (i.e., the magnitude of the energy splitting between the two states and the slopes of the two avoiding curves) of the avoided crossings in a stabilization graph to compute the lifetimes of the metastable states. Basically, the larger the avoided crossing energy splitting between the two states, the shorter is the lifetime of the resonance state.

Acknowledgments: This work was supported by NSF grants \#9982420 and \#0240387. 


\section{References}

${ }^{1}$ J. C. Rienstra-Kiracofe, G. S. Tschumper, H. F. Schaefer, III, S. Nandi, and G. B. Ellison, Acc. Chem. Research, 102, 231-282 (2002).

${ }^{2}$ G. Herzberg, Molecular Spectra and Molecular Structure I. p. 512, Van Nostrand, New York (1950).

${ }^{3}$ G. I. Gutsev and A. I. Boldyrev, The Theoretical Investigation of the Electron Affinity of Chemical Compounds, Adv. Chem. Phys. 1985, 61, 169-221.

J. Baker, R. H. Nobes, and L. Radom, The Evaluation of Molecular Electron Affinities, J. Comp. Chem. 1986, 7, 349-358.

J. Simons and K. D. Jordan, Ab Initio Electronic Structure of Anions, Chem. Rev. 1987, $87,535-555$.

J. Kalcher and A. F. Sax, Gas Phase Stabilities of Small Anions--Theory and Experiment in Cooperation, Chem. Rev. 1994, 94, 2291-2318; J. Kalcher, Gas-Phase Stabilities of Small Anions, J. Ann. Reports, Sec. C, Royal Soc. of Chem. 1996, 93, 147-186.

R. S. Berry, Small Free Negative Ion, Chem. Rev. 1969, 69, 533-542.

The most up to date overview is given in ref. 1

4 T. H. Dunning and V. McKoy, J. Chem. Phys. 47, 1735 (1967); J. Chem. Phys. 48, 5263 (1968); T. I. Shibuya and V. McKoy, J. Chem. Phys. 53, 2208 (1970)

${ }^{5}$ D. J. Rowe, Rev. Mod. Phys. 40, 153 (1968); D. J. Rowe, Nuclear Collective MotionModels and Theory, Methuen, and Co., London (1970).

${ }^{6}$ J. Simons and W. D. Smith, "Theory of Electron Affinities of Small Molecules", J. Chem. Phys. 58, 4899 (1973).

${ }^{7}$ J. Simons and P. Jørgensen, J. Chem. Phys. $\underline{64} 1413$ (1976); Tsung-Tai Chen, Jack Simons and K. D. Jordan, Chem. Phys. 14, 145 (1976); Jack Simons, J. Chem. Phys. $\underline{64}$, 4541 (1976).

J. Simons, Int. J. Quant. Chem. XII, 227 (1977); E. Dalgaard and J. Simons, J. Phys. B: Atom. Molec. Phys. 10, 2767 (1977); J. Simons, Ann. Rev. Phys. Chem. 28, 15 (1977); A. Banerjee, R. Shepard, and J. Simons, Int. J. Quant. Chem: Quantum Chemistry 
Symposium 12, 389 (1978); R. A. Donnelly and J. Simons, J. Chem. Phys. 73, 2858 (1980).

${ }^{8}$ See, for example, Second Quantization-Based Methods in Quantum Chemistry, by P. Jørgensen and J. Simons, Academic Press (1981).

${ }^{9}$ R. Manne, Chem. Phys. Lett. 45, 470 (1977).

${ }^{10}$ The first- and second-order density matrices, respectively, have elements given by $\left\langle 0, \mathrm{~N}\left|\mathrm{j}^{+} \mathrm{k}\right| 0, \mathrm{~N}\right\rangle$ and $\left\langle 0, \mathrm{~N}\left|\mathrm{j}^{+} \mathrm{k}^{+} \mathrm{lh}\right| 0, \mathrm{~N}\right\rangle$.

${ }^{11}$ J. Simons, T. -T. Chen, and W. D. Smith, Chem. Phys. Lett. 26, 296 (1974); W. D. Smith, T. -T. Chen and J. Simons, Chem. Phys. Lett. 27, 499 (1974); Karen M. Griffing and Jack Simons, J. Chem. Phys. 62, 535 (1975); J. Kenney and J. Simons, J. Chem. Phys. 62, 592 (1975); K. Griffing, J. Kenney, J. Simons and K. Jordan, J. Chem. Phys. 63, 4073 (1975); Karen Griffing and Jack Simons, J. Chem. Phys. 64, 3610 (1976); K. D. Jordan, K. M. Griffing, J. Kenney, E. L. Andersen and J. Simons, J. Chem. Phys. 64, 4730 (1976); E. Andersen and J. Simons, J. Chem. Phys. 64, 4548 (1976); K. D. Jordan and J. Simons, J. Chem. Phys. 65, 1601 (1976); E. Andersen and J. Simons, J. Chem. Phys. 65, 5393 (1976); E. Andersen and J. Simons, J. Chem. Phys. 66, 2427 (1977); J. Simons, Ann. Rev. Phys. Chem. 28, 15 (1977).

12 "A Complete Treatment of the Electron Propagator Through Third Order", Poul Jørgensen and Jack Simons, J. Chem. Phys. 63, 5302 (1975).

${ }^{13}$ J. Linderberg and Y. Öhrn, Propagators in Quantum Chemistry, Academic Press, London (1973)

${ }^{14}$ L.S. Cederbaum, G. Holneicher, and S. Peyerimhoff, Chem. Phys. Lett. 11421 (1971);

L.S. Cederbaum, G. Holneicher, and W. von Niessen, Chem. Phys. Lett. 18, 503 (1973);

L. S. Cederbaum, Theo. Chim. Acta 31, 239 (1973).

${ }^{15}$ J. D. Doll and W. P. Reinhardt, J. Chem. Phys. 67, 1169 (1972).

${ }^{16}$ B. S. Yarlagadda, Gy. Csanak, H. S. Taylor, B. Schneider, and R. Yaris, Phys. Rev. A7, 146 (1973).

${ }^{17}$ B. T. Pickup and O. Goscinski, Mol. Phys. 36, 1013 (1973).

${ }^{18}$ G. D. Purvis and Y. Öhrn, J. Chem. Phys. 60, 4063 (1974); 62, 2045 (1975). 
${ }^{19}$ See, for example, J. V. Ortiz, Leszczynski, J. (Ed) Computational Chemistry: Reviews of Current Trends (Singapore: World Scientific) Vol 2 pp 1-61(1997)

${ }^{20}$ M. J. Frisch, G. W. Trucks, H. B. Schlegel, G. E. Scuseria, M. A. Robb, J. R. Cheeseman, J. A.Montgomery, Jr., T. Vreven, K. N. Kudin, J. C. Burant, J. M. Millam, S. S. Iyengar, J. Tomasi, V. Barone, B. Mennucci, M. Cossi, G. Scalmani, N. Rega, G. A. Petersson, H. Nakatsuji, M. Hada, M. Ehara, K. Toyota, R. Fukuda, J. Hasegawa, M. Ishida, T. Nakajima, Y. Honda, O. Kitao, H. Nakai, M. Klene, X. Li, J. E. Knox, H. P. Hratchian, J. B. Cross, C. Adamo, J. Jaramillo, R. Gomperts, R. E. Stratmann, O. Yazyev, A. J. Austin, R. Cammi, C. Pomelli, J. W. Ochterski, P. Y. Ayala, K. Morokuma, G. A. Voth, P. Salvador, J. J. Dannenberg, V. G. Zakrzewski, S. Dapprich, A. D. Daniels, M. C. Strain, O. Farkas, D. K. Malick, A. D. Rabuck, K. Raghavachari, J. B. Foresman, J. V. Ortiz, Q. Cui, A. G. Baboul, S. Clifford, J. Cioslowski, B. B. Stefanov, G. Liu, A. Liashenko, P. Piskorz, I. Komaromi, R. L. Martin, D. J. Fox, T. Keith, M. A. Al-Laham, C. Y. Peng, A. Nanayakkara, M. Challacombe, P. M.W. Gill, B. Johnson, W. Chen, M. W. Wong, C. Gonzalez, and J. A. Pople, Gaussian, Inc., Pittsburgh PA, 2003.

J. V. Ortiz, Toward an Exact One-Electron Picture of Chemical Bonding, Advances in Quantum Chemistry 35, 33-52 (1999).

Propagating Insight: A Tribute to the Works of Yngve Öhrn J. V. Ortiz and H. A. Kurtz, Editors, Advances in Quantum Chemistry 35, Academic Press, New York, 1999.

${ }^{21}$ M. M. Morrel, R. G. Parr, and M. Levy, J. Chem. Phys. 62, 549 (1975).

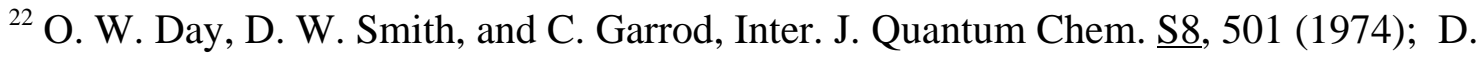

W. Smith and O. W. Day, J. Chem. Phys. 62, 113 (1975).

${ }^{23}$ E. Andersen and J. Simons, J. Chem. Phys. 66, 1067 (1977).

${ }^{24}$ J. Cioslowski, P. Piskorz, and G. Liu, J. Chem. Phys. 107, 6804 (1997).

${ }^{25}$ A. Banerjee, R. Shepard, and J. Simons, "One-Particle Green's Function with Multiconfiguration Reference States", Int. J. Quant. Chem: Quantum Chemistry Symposium 12, 389 (1978). 
${ }^{26}$ H. J. Monkhorst, Int. J. Quantum Chem. Symp., 11, 421 (1977); M. Nooijen and R. J. Bartlett, J. Chem. Phys., 102, 3629 (1995). Equation-of-motion coupled-cluster method for electron attachment;

D. Mukhopadhyay, S. Mukhopadhyay, R. Chaudhuri, and D. Mukherjee, Theor. Chim. Acta, 80, 441 (1991). Aspects of separability in the coupled cluster based direct method for energy differences;

J. F. Stanton and R. J. Bartlett, J. Chem. Phys., 98, 7029 (1993). The equation of motion coupled-cluster method. A systematic biorthogonal approach to molecular excitation energies, transition probabilities, and excited state properties.

${ }^{27}$ R. J. Bartlett and J. F. Stanton, in Reviews in Computational Chemistry, edited by K. B. Lipkowitz and D. B. Boyd VCH, New York, 1994, Vol.5.

${ }^{28}$ A. U. Hazi and H. S. Taylor, Phys. Rev. A1, 1109 (1970).

${ }^{29}$ J. Simons, J. Chem. Phys. 75, 2465 (1981); R. F. Frey and J. Simons, J. Chem. Phys. 84, 4462 (1986). 